\title{
Neoadjuvant therapy for locally advanced melanoma: new strategies with targeted therapies
}

This article was published in the following Dove Press journal:

OncoTargets and Therapy

19 June 2014

Number of times this article has been viewed

\section{Michele La Greca' \\ Giuseppe Grasso ${ }^{2}$ \\ Giovanna Antonelli' \\ Alessia Erika Russo' \\ Salvatore Bartolotta ${ }^{3}$ \\ Alessandro D’Angelo' \\ Felice Vito Vitale' \\ Francesco Ferraù'}

'Medical Oncology Department, San Vincenzo Hospital, Taormina, Messina, Italy; ${ }^{2}$ Pathology Department, San

Vincenzo Hospital, Taormina, Messina, Italy; ${ }^{3}$ Surgical Unit, Casa di Cura

Gretter-Lucina, Catania, Catania, Italy
Correspondence: Francesco Ferraù Medical Oncology Department, San Vincenzo Hospital, Contrada Sirina, 98039 Taormina (ME), Italy Tel +390942579282

Fax +39094252215

Email francescoferrau@tin.it
Abstract: Neoadjuvant chemotherapy has been successfully tested in several bulky solid tumors, but it has not been utilized in advanced cutaneous melanoma, primarily because effective medical treatments for this disease have been lacking. However, with the development of new immunotherapies (monoclonal antibodies specific for cytotoxic $\mathrm{T}$ lymphocyte-associated antigen 4 [anti-CTLA-4] and programmed death protein-1 [anti-PD1]) and small molecules interfering with intracellular pathways (anti-BRAF and mitogen-activated protein kinase kinase [anti- MEK]) the use of this approach is becoming a viable treatment strategy for locally advanced melanoma. The neoadjuvant setting provides a double opportunity for a better knowledge of these drugs: a short-term evaluation of their intrinsic activity, and a deeper analysis of their action and resistance-induction mechanisms. BRAF inhibitors seem to be ideal candidates for the neoadjuvant setting, because of their prompt, repeatedly confirmed response in $\mathrm{V} 600 \mathrm{E}$ $B R A F$-mutant metastatic melanoma. In this report we summarize studies focused on the neoadjuvant use of traditional medical treatments in advanced melanoma and anecdotal cases of this approach with the use of biologic therapies. Moreover, we discuss our experience with neoadjuvant targeted therapy as a priming for radical surgery in a patient with $B R A F$ V600E mutation-positive advanced melanoma.

Keywords: neoadjuvant setting, biologic, targeted therapy, vemurafenib, advanced melanoma

\section{Introduction}

Lymphotropism related to cutaneous melanoma is typically treated with therapeutic strategies such as sentinel lymph node biopsy or radical dissection of the lymph node basin. However, macroscopically radical surgery, usually considered an elective therapeutic choice, can be difficult because of lymph node position or size; in this case, an effective medical treatment prior to surgery is desirable in order to treat the initially unresectable disease. This strategy has been successfully tested in several solid tumors, such as head and neck, breast, and lung carcinomas, but it has not been utilized in advanced cutaneous melanoma, primarily because effective medical treatments for this disease have been lacking.

Great expectations are nowadays placed on biologic therapies, especially $B R A F$ inhibitors, because of their high and reproducible activity against V600E $B R A F$-mutant advanced or metastatic melanoma.

In this report, we discuss our experience with neoadjuvant targeted therapy as a priming for radical surgery in a patient with $B R A F \mathrm{~V} 600 \mathrm{E}$ mutation-positive advanced melanoma. 


\section{Case report}

A 42-year-old male, after surgical exeresis of a pigmented lesion on the anterior abdominal wall, received a diagnosis of "nodular cutaneous melanoma, mainly composed of epithelioid cells, vertical phase of growth; Breslow thickness: 15.38 mm, Clark level: IV, Mib-1 >40\%." Positron emission tomography (PET) and thoracoabdominal computed tomography (CT) staging revealed abnormal uptake and enlargement at the level of the right inguinal, iliac, and obturator lymph nodes.

Radical resection of these synchronous iuxtaregional lymph node metastases was judged to be impracticable due to deep localization and an extensive growth pattern. Therefore, the patient began chemotherapy treatment with a cisplatin-vindesine-dacarbazine regimen and completed three cycles with reasonable tolerability. CT re-evaluation showed stabilization of disease, so two additional cycles of the same chemotherapy were given; mutational analysis of $B R A F$ showed presence of $\mathrm{V} 600 \mathrm{E}$ mutation.

After the fifth chemotherapy cycle, further evaluations showed increase in the diameters of the right inguinal, iliac, and obturator lymph nodes (CT scan, Figure 1) and abnormal fluorodeoxyglucose uptake in the same sites. The patient complained of oppressive pain in the right inguinocrural and pelvic regions and was treated with transdermal fentanyl (50 mcg over 72 hours) and acetaminophen (1,000 mg twice daily) and was started on vemurafenib treatment (960 mg twice daily). After the first week of treatment, a prompt improvement in pain (a reduction from 8 to 3 on a $0-10$ numerical rating scale) was registered. Medical treatment was very well tolerated, with rapid weaning from the analgesics. After the first month of vemurafenib treatment, evaluation with PET and CT scan revealed volume reduction and metabolic silencing of pelvic and inguinal adenopathies (Figure 2). The patient thus continued treatment with full-dose vemurafenib, without dose reductions or delays, along with monthly hematological checking and quarterly $\mathrm{CT}$ and PET scans.

After 10 months of vemurafenib treatment, radiographic control showed complete morphological and metabolic remission of disease (Figure 3). After multidisciplinary evaluation, surgical exeresis of affected lymph node sites was planned, on the basis of three considerations: 1) lymph nodes were the only site of disease since diagnosis and after therapy; 2) in these sites, melanoma showed marked volumetric reduction and complete metabolic silencing; and 3) 10 months is longer than the median duration of objective responses to vemurafenib (usually 6-8 months).

Right inguino-iliac-obturator lymph node dissection was performed with the following histological response: "metastasis from epithelioid melanoma, intensely pigmented in three on 18 inguinal lymph nodes; no metastatic deposits in remaining 25 external iliac and obturator lymph nodes." As a precaution, vemurafenib was suspended 2 days before surgery and restarted 2 days afterward.

Five months after surgery, the patient is continuing with vemurafenib therapy, requiring no analgesics, and reporting neither wound problems nor other side effects.

\section{Neoadjuvant treatment in locally advanced melanoma: premises}

The development of new immunotherapies (monoclonal antibodies specific for cytotoxic $\mathrm{T}$ lymphocyte-associated

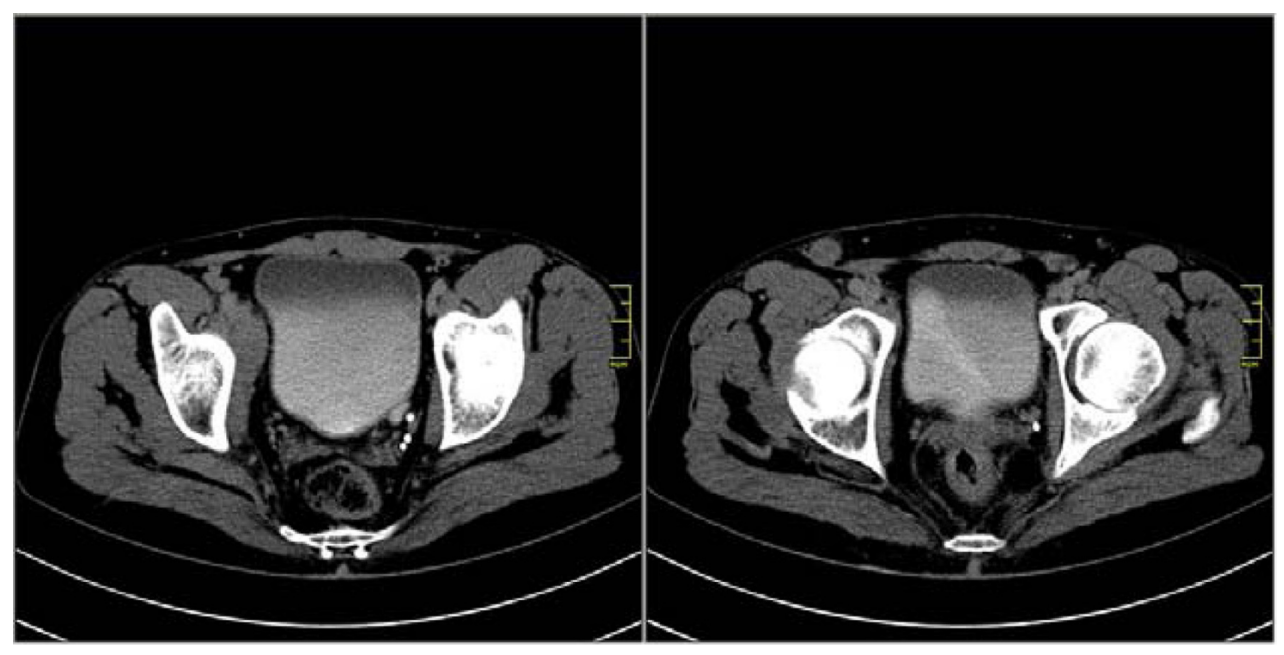

Figure I CT scan, October 2012 (before vemurafenib therapy). Abbreviation: $\mathrm{CT}$, computed tomography. 


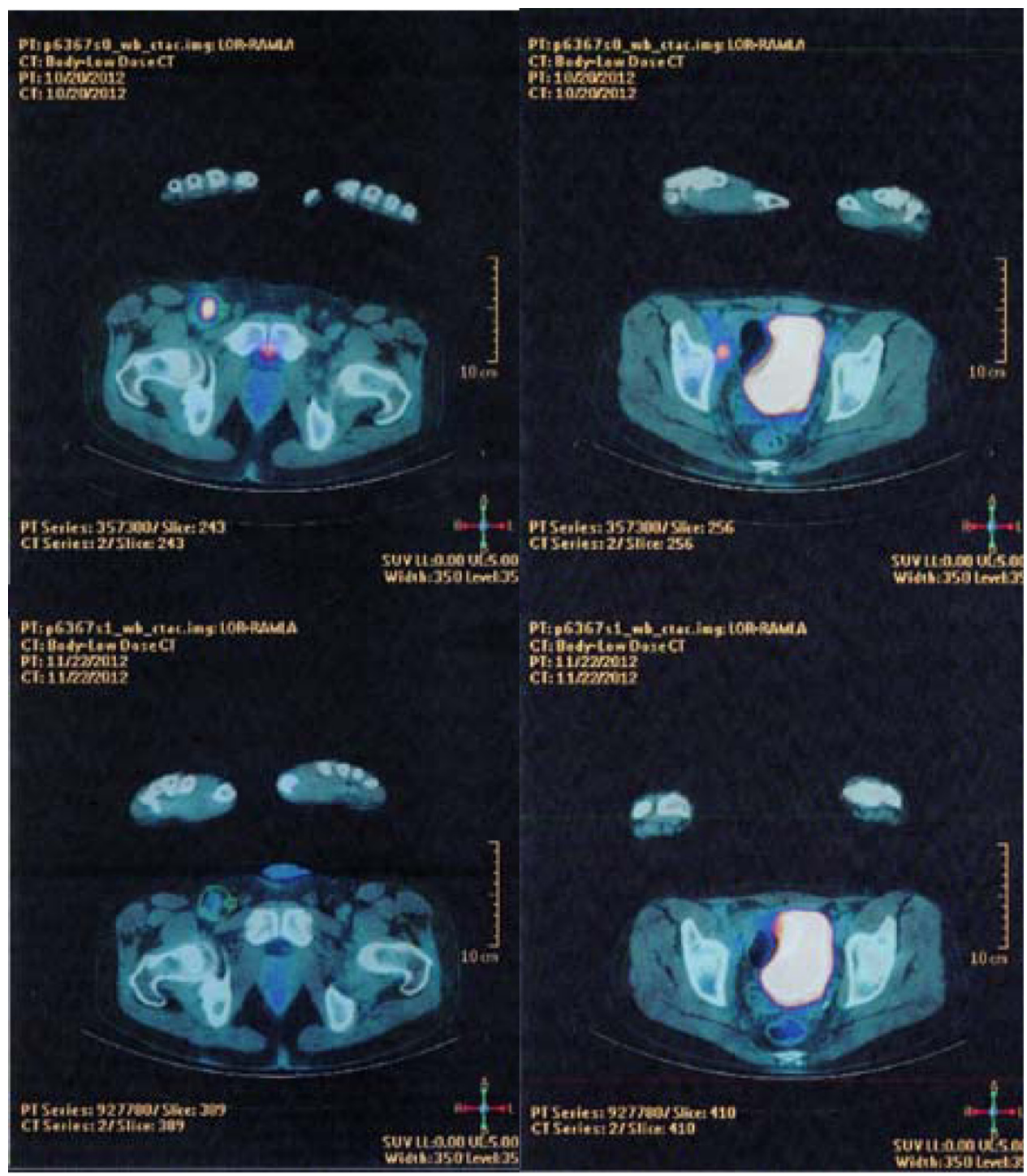

Figure 2 Comparison between PET at baseline (upper row) and PET after I month of vemurafenib therapy (lower row).

Abbreviation: PET, positron emission tomography.

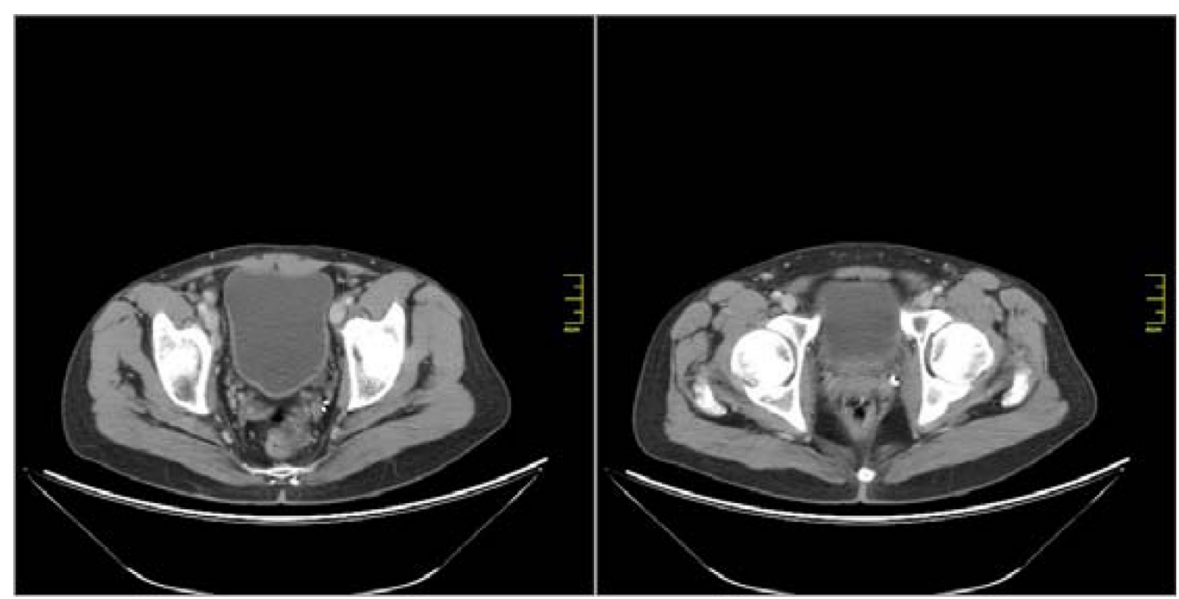

Figure 3 CT scan, July 2013 , after 10 months of vemurafenib therapy.

Note: Imaging shows near-complete resolution of previously enlarged pelvic and inguinal nodes.

Abbreviation: $\mathrm{CT}$, computed tomography. 
antigen 4 [anti-CTLA-4] and programmed death protein-1 [anti-PD1]) and small molecules interfering with intracellular pathways (anti-BRAF, anti-mitogen-activated protein kinase kinase [MEK]) is expected to revolutionize medical treatment of advanced cutaneous melanoma (Table 1). These molecules are being evaluated through a series of recently begun adjuvant trials whose results will not be available for several years. ${ }^{1}$ Neoadjuvant treatment settings provide, instead, a double opportunity for a better knowledge of these drugs: a short-term evaluation of their intrinsic activity, and a deeper analysis of their action and resistance-induction mechanisms. $^{2}$

Chemotherapeutic or biochemotherapeutic approaches to locally advanced melanoma have been considered as a therapeutic modality capable of improving overall prognosis, rather than aiming to improve the rate of resectability, as in other solid tumors.

\section{Traditional medical treatments (immunotherapies; chemotherapy; others)}

Early on in the treatment of melanoma, historically available therapeutic choices, such as chemotherapy and immunotherapy with interferon or interleukin-2, were employed. The literature does not provide relevant information in this field, either because of insufficient sample sizes or poor quality of evidence. ${ }^{3}$

For example, the largest study using interferon in the neoadjuvant setting examined only 17 patients with stage 3 melanoma; the American high dose schedule was provided ( $20 \mathrm{mg} / \mathrm{m}^{2}, 5$ days per week for 4 weeks), followed by surgery.
The results were relevant, with a $55 \%$ objective response rate and $15 \%$ pathological complete remission; however, technical difficulties and severe side effects precluded further study with this schedule. ${ }^{4}$

The largest neoadjuvant therapy trial was conducted in 92 patients with stage 3 melanoma, histologically proved by sentinel node biopsy (42 patients) or clinically apparent lymphadenopathy biopsy (50 patients), using a "sandwich" biochemotherapy schedule. Treatment consisted of two preoperative and two postsurgical cycles with a regimen of cisplatin, $20 \mathrm{mg} / \mathrm{m}^{2}$, days $1-4$; dacarbazine $800 \mathrm{mg} / \mathrm{m}^{2}$, day 1 ; and vinblastine $1.6 \mathrm{mg} / \mathrm{m}^{2}$, days $1-4$. This regimen was augmented with interleukin-2 $\left(36 \mathrm{MU} / \mathrm{m}^{2}\right.$ total dose over 4 days) and alpha-interferon (5 MU/m², days 1-5); granulocyte-colony stimulating factor was associated from the outset. Of the 50 patients with measurable disease, $26 \%$ achieved objective responses and 4\% achieved complete remission; at median 40 months follow-up, relapsefree survival and overall survival rates were $64 \%$ and $78 \%$, respectively. The prevalence of grade 3-4 toxicities, reported as "manageable" by the authors, was indeed significant: neutropenia in $77 \%$ of patients, emesis in $20 \%$, and electrolytic imbalances in $15 \% .^{5}$

This work duplicated a previous pilot study in 64 patients with the same subset of disease and the same biochemotherapeutic schedule; overall response rate was $50 \%$, with four (6.5\%) complete pathological responses, mainly related to a lesser initial tumor burden. ${ }^{6}$

Early activity demonstrated by biochemotherapy in Phase II studies of metastatic melanoma was not confirmed in two wide randomized Phase III studies comparing

Table I Cytotoxic and biologic agents against malignant melanoma

\begin{tabular}{|c|c|c|}
\hline Drug & Structure & Mechanism(s) of action \\
\hline Alpha-interferon & Glycoprotein & Antiviral, immunomodulatory, and antiproliferative effects \\
\hline \multirow[t]{3}{*}{ Interleukin-2 } & \multirow[t]{3}{*}{ Glycoprotein } & Regulating activated T-cell proliferation \\
\hline & & Promoting regulatory T-cell development, survival and maintenance \\
\hline & & Promoting B-cell proliferation and immunoglobulin production \\
\hline Temozolomide & Organic polycyclic compound containing & Cytotoxic action by DNA alkylation \\
\hline & an imidazole ring fused to a tetrazine ring & \\
\hline Bleomycin & Glycopeptide antibiotic & Cytotoxic action by induction of DNA strand breaks \\
\hline Ipilimumab & Humanized lgG monoclonal antibody & $\begin{array}{l}\text { Overcoming CTLA-4-mediated T-cell suppression to enhance the } \\
\text { immune response against tumors }\end{array}$ \\
\hline Vemurafenib, dabrafenib & Synthetic small molecules & Binding to the ATP-binding site of BRAF (V600E) kinase with \\
\hline & & inhibition of downstream pathway \\
\hline Trametinib & Synthetic small molecule & Inhibition of MEK I and 2 signaling \\
\hline Nivolumab, lambrolizumab & Humanized lgG monoclonal antibodies & $\begin{array}{l}\text { Binding to PD-I resulting in enhancement of the immune response } \\
\text { against tumors }\end{array}$ \\
\hline MPDL3280A & Humanized IgG monoclonal antibody & Binding to PD-LI and inhibition of its receptor, PD-I \\
\hline
\end{tabular}

Abbreviations: ATP, adenosine triphosphate; CTLA-4, cytotoxic T-lymphocyte antigen-4; MEK, mitogen-activated protein kinase kinase; PD-I, programmed cell death protein-I; PD-LI, programmed cell death protein ligand-I. 
biochemotherapy with monochemotherapy. ${ }^{7,8}$ On the basis of these poor results, and in consideration of the growing rate of severe adverse events related to biochemotherapy, the possible role of biochemotherapy in a neoadjuvant setting for locally advanced melanoma has not been further explored in randomized studies.

A 1996 study from Yale University was based on a different rationale: 16 patients with metastatic melanoma were subjected to surgery for residual disease after induction chemotherapy; visceral metastases, except brain and bones, were included. In historical comparison with a subset of patients with similar cancer stage but treated with chemotherapy only, a clear benefit emerged from the inclusion of surgery following chemotherapeutic induction. ${ }^{9}$

It should be noted that presurgical biochemotherapy does not seem to increase surgical complications, at either the cutaneous or nodal level. ${ }^{10} \mathrm{~A}$ favorable response to primary chemotherapy has also been proposed as a selection criterion for candidate patients for metastasectomy, with complete pathological response being a predictor of longterm survival. ${ }^{11}$

Temozolomide activity has been tested in a small series of studies in patients with locally advanced melanoma. Nineteen patients with operable lymph node metastases (14 stage 3 , five stage $4 \mathrm{M} 1 \mathrm{a}$ ) were treated with temozolomide in the neoadjuvant setting $\left(75 \mathrm{mg} / \mathrm{m}^{2}\right.$ daily for 6 weeks, followed by 2 weeks of rest) and subsequent surgery. The objective response rate was $16 \%$ with two complete responses, not linked to tumor O-6-methylguanine-DNA methyltransferase promoter mutational status, similar to results obtainable in metastatic melanoma. $^{12}$

A Mayo Clinic study examined preoperative chemotherapy in vulvar and vaginal melanoma. A female with recurrent melanoma was treated with temozolomide followed by pelvic exenteration; she died within 3 months. Two other patients with recurrent melanoma were treated with primary chemotherapy consisting of carboplatin, paclitaxel, and bevacizumab followed by radical surgery, and finally treated with the same chemotherapeutic schedule in adjuvant setting; both the patients are alive and free of disease at 5 years and 3 years, respectively. ${ }^{13}$

In light of the overall modest activity of chemotherapy in melanoma, alternative therapeutic choices have been explored in the neoadjuvant stage, such as electrochemotherapy and radiotherapy. Electrochemotherapy with bleomycin has been tested in order to improve surgical outcomes; an Italian case report of scalp melanoma that recurred in the cheek reported optimal results from preoperative electrochemotherapy in term of tolerability, feasibility, and cosmetic effects. ${ }^{14}$ In 12 patients with histologically confirmed stage 3 melanoma, preoperative radiotherapy was administered at a maximum dose of 48 Gy in 20 fractions. Two patients developed minor surgical complications and the 1-year in-field control rate was $92 \%$; this strategy was suggested particularly for largevolume disease. ${ }^{15}$

\section{Innovative medical treatment: targeted therapies}

Literature reports regarding biologic and targeted therapies are inevitably anecdotal. Only early indications concerning the role of new biologic therapy in neoadjuvant setting can be found.

An exploratory trial using preoperative ipilimumab in histologically proved, locally advanced melanoma is in progress at the University of Pittsburgh. After biopsy, two doses of ipilimumab $10 \mathrm{mg} / \mathrm{kg}$ were administered every three weeks, followed by radical lymphadenectomy; 1 month after surgery, ipilimumab treatment was resumed for two additional cycles. Preliminary results presented at the American Society of Clinical Oncology 2012 Annual Meeting showed median progression-free survival of 15.5 months and a probability of progression-free survival of $82 \%$ and $53 \%$ at 6 and 12 months, respectively. The study included a correlated serological and histopathological substudy in order to characterize the tumor microenvironment and pre- and post-ipilimumab immunological modifications. ${ }^{16}$

Ipilimumab's mechanism of action and pattern of time response, with typically rare objective responses and longterm disease stabilization, make it less effective for neoadjuvant use, where a higher response rate and rapid tumor shrinking are critical. In this sense, $B R A F$ inhibitors seem to be ideal candidates because of their prompt, repeatedly confirmed response in $\mathrm{V} 600 \mathrm{E} B R A F$-mutant metastatic melanoma. Some literature reports seem to confirm these theoretical premises.

Vemurafenib was successfully employed in a young patient with wide brain melanoma metastases, in an example of multifocal disease previously surgically treated for digiunal metastases and treated with ipilimumab. Full-dose vemurafenib obtained resolution of left hemiparesis at day 4; after 37 days of therapy, tumor shrinkage $(>50 \%)$ allowed macroscopically complete brain metastasectomy, followed by stereotactic radiotherapy and vemurafenib restarting at the seventh day postsurgery. Neither melanoma relapse nor relevant adverse events were registered in the following 
12 months of vemurafenib treatment. This report evidences: 1) the activity of vemurafenib in a tricky disease site such as brain metastasis, even in a patient pretreated with ipilimumab; 2) a prolonged complete clinical response ( $>1$ year); and 3 ) the feasibility of vemurafenib in close preoperative and postoperative timing, without negative wound effects. ${ }^{17}$

In another case report, preoperative vemurafenib treatment was employed in a patient with axillary, fixed bulky nodal disease from primary unknown melanoma. After 4 months of $B R A F$ inhibitor treatment, the axillary mass dimension shrank from $15 \mathrm{~cm}$ to $8 \mathrm{~cm}$, allowing detachment from the chest wall in subsequent ablative surgery with myocutaneous autograft. The patient was disease-free 5 months after surgery, having continued vemurafenib treatment during this time. ${ }^{18}$

A bulky axillary and laterocervical recurrence from cutaneous elbow melanoma was brought back to surgical suitability (axillary and modified neck dissection) after 4 months of vemurafenib treatment, obtaining an objective response $>50 \%$; vemurafenib was then resumed and continued for 6 months without recurrence. ${ }^{19}$

These early studies illustrate that vemurafenib in the melanoma neoadjuvant setting produced a prompt objective rate difficult to imagine with previous traditional therapy in locally advanced cutaneous melanoma.

\section{Conclusion}

Locally advanced or metastatic melanoma is probably turning from an orphan, medically optionless disease into a modern laboratory for clinical research, in which there is the possibility of successfully experimenting with the newest therapeutic biological weapons. This revolutionary change in clinical perspectives is primarily sustained by the suitability of new biologic drugs, where $B R A F$ inhibitors represent the first-line molecules.

Their use, targeted on the basis of selective molecular characteristics, and their optimal tolerability are outlining a substantially new scenario, in which a response rate thus far unthinkable in melanoma can be easily be achieved with biochemotherapy and subsequent surgery.

\section{Disclosure}

The authors report no conflicts of interest in this work.

\section{References}

1. Tarhini AA, Pahuja S, Kirkwood JM. Neoadjuvant therapy for high-risk bulky regional melanoma. $J$ Surg Oncol. 2011;104(4):386-390.
2. Davar D, Tarhini AA, Kirkwood JM. Adjuvant immunotherapy of melanoma and development of new approaches using the neoadjuvant approach. Clin Dermatol. 2013;31(3):237-250.

3. Sarnaik AA, Zager JS, Sondak VK. Multidisciplinary management of special melanoma situations: oligometastatic disease and bulky nodal sites. Curr Oncol Rep. 2007;9(5):417-427.

4. Moschos SJ, Edington HD, Land SR, et al. Neoadjuvant treatment of regional stage IIIB melanoma with high-dose interferon alfa- $2 \mathrm{~b}$ induces objective tumor regression in association with modulation of tumor infiltrating host cellular immune responses. J Clin Oncol. 2006;24(19): 3164-3171.

5. Lewis KD, Robinson WA, McCarter M, et al. Phase II multicenter study of neoadjuvant biochemotherapy for patients with stage III malignant melanoma. J Clin Oncol. 2006;24(19):3157-3163.

6. Buzaid AC, Colome M, Bedikian A, et al. Phase II study of neoadjuvant concurrent biochemotherapy in melanoma patients with local-regional metastases. Melanoma Res. 1998;8(6):549-556.

7. Atkins MB, Hsu J, Lee S, et al; Eastern Cooperative Oncology Group. Phase III trial comparing concurrent biochemotherapy with cisplatin, vinblastine, dacarbazine, interleukin-2, and interferon alfa- $2 \mathrm{~b}$ with cisplatin, vinblastine, and dacarbazine alone in patients with metastatic malignant melanoma (E3695): a trial coordinated by the Eastern Cooperative Oncology Group. J Clin Oncol. 2008;26(35):5748-5754.

8. Keilholz U, Punt CJ, Gore M, et al. Dacarbazine, cisplatin, and interferon-alfa- $2 \mathrm{~b}$ with or without interleukin-2 in metastatic melanoma: a randomized phase III trial (18951) of the European Organisation for Research and Treatment of Cancer Melanoma Group. J Clin Oncol. 2005;23(27):6747-6755.

9. Sasson HN, Poo WJ, Bakas MH, Ariyan S. Prolonged survival in patients with advanced melanoma treated with neoadjuvant chemotherapy followed by resection. Ann Plast Surg. 1996;37(3):286-292.

10. Kounalakis N, Gao D, Gonzalez R, et al. A neoadjuvant biochemotherapy approach to stage III melanoma: analysis of surgical outcomes. Immunotherapy. 2012;4(7):679-686.

11. Jouary T, Legros C, Lalanne N, et al. Neoadjuvant chemotherapy: a new criterion for selection of candidate patients for surgery of low tumour burden metastases from malignant melanoma? Br J Dermatol. 2010;163(1):183-187.

12. Shah GD, Socci ND, Gold JS, et al. Phase II trial of neoadjuvant temozolomide in resectable melanoma patients. Ann Oncol. 2010;21(8): $1718-1722$.

13. Janco JM, Markovic SN, Weaver AL, Cliby WA. Vulvar and vaginal melanoma: case series and review of current management options including neoadjuvant chemotherapy. Gynecol Oncol. 2013;129(3): 533-537.

14. Mozzillo N, Caracò C, Mori S, et al. Use of neoadjuvant electrochemotherapy to treat a large metastatic lesion of the cheek in a patient with melanoma. J Transl Med. 2012;10:131.

15. Foote M, Burmeister B, Dwyer P, et al. An innovative approach for locally advanced stage III cutaneous melanoma: radiotherapy, followed by nodal dissection. Melanoma Res. 2012;22(3):257-262.

16. Tarhini AA, Edington $\mathrm{H}$, Butterfield $\mathrm{LH}$, et al. Immune monitoring of the circulation and the tumor microenvironment in patients with regionally advanced melanoma receiving neoadjuvant ipilimumab. PLoS One. 2014;9(2):e87705.

17. Kolar GR, Miller-Thomas MM, Schmidt RE, Simpson JR, Rich KM, Linette GP. Neoadjuvant treatment of a solitary melanoma brain metastasis with vemurafenib. J Clin Oncol. 2013;31(3):e40-e43.

18. Koers K, Francken AB, Haanen JB, Woerdeman LA, van der Hage JA. Vemurafenib as neoadjuvant treatment for unresectable regional metastatic melanoma. J Clin Oncol. 2013;31(16):e251-e253.

19. Fadaki N, Cardona-Huerta S, Martineau L, et al. Inoperable bulky melanoma responds to neoadjuvant therapy with vemurafenib. $B M J$ Case Rep. 2012;2012. 
OncoTargets and Therapy

\section{Publish your work in this journal}

OncoTargets and Therapy is an international, peer-reviewed, open access journal focusing on the pathological basis of all cancers, potential targets for therapy and treatment protocols employed to improve the management of cancer patients. The journal also focuses on the impact of management programs and new therapeutic agents and protocols on

patient perspectives such as quality of life, adherence and satisfaction. The manuscript management system is completely online and includes a very quick and fair peer-review system, which is all easy to use. Visit http://www.dovepress.com/testimonials.php to read real quotes from published authors.

Submit your manuscript here: http://www.dovepress.com/oncotargets-and-therapy-journal 